\title{
How conventional Flight-Test Instrumentation concepts can contribute to the innovative eVTOL aerospace development
}

\author{
Luis Antonio Jacob da Motta \\ Lilium GmbH, Weßling - Germany \\ luis.motta@lilium.com
}

\begin{abstract}
:
Even in face of all innovative aspects around VTOL aircraft development, specially for all-electric airplanes, the conventional FTI concepts shows up your real value: from Processes Organization until Test Results, FTI can contribute providing solution in many scopes of the Flight-Testing, what includes Requirements Control, Data Acquisition and Special Systems Design, Flight-Test Data Processing and Analysis and Flight Results Reports. In this article, we'll describe how the conventional FTI is being developed and implemented from the scratch in an eVTOL Startup, its development challenges and how to keep improving the instrumentation concepts in front of all new technologies demand for manned and unmanned flights.
\end{abstract}

Key words: Data Acquisition, Flight-Testing, VTOL, Data Processing, Startup.

\section{Introduction}

Keeping in mind we are planning to validate something complete new and challenger, and doesn't matter what you are planning to test in the air, the first and mainly concern we must have "on the table" when designing is SAFETY. And having it as our primary requirement in the new project, now we can focus in everything we must comply with all engineering requirements, combining them between each other, providing us coverage around the second most import aspect in aviation: RELIABILITY and CONTROLABILITY.

In that way the Flight-Test Instrumentation (FTI) has and important involvement and contributes providing all relevant measurements and monitoring systems to the Systems Engineering and Flight-Test Crew during performing flight-tests. The idea is to have the clear aircraft's behavior when performing the tests (Real-Time Data Processing), minimizing risks and maximizing performance, and the possibility to analyze deeply all data and flight conditions after tests (Post-Flight Data Processing).

Even having an innovative scenario in this allelectric aircraft development, the conventional FTI shows how important, reliable and useful it is during the Flight-Test Campaigns (FTC), of course respecting and complying with all electrical related aspects like Electrical Magnetic Interference (EMI) and Unmanned Flight Operation characteristics and restrictions.

\section{FTI Macro Process}

Represents a group of best practices sequence of development phases that the instrumentation is being performed at Lilium for a better and faster implementation:

- Conception:

Consists of phase where all preliminary FTI definitions are evaluated;

- Project:

Consists of phase where the FTI System is fully documented, which includes electrical and mechanical installation drawings;

- Integration:

Consists of phase where the proposed FTI System, sensors calibration and aircraft system integration is performed;

- Software Development:

Consists of phase where all necessary software (SW) development for controlling $\mathrm{FTI}$ information and Data Processing is performed (continuous development), even along the FTC's;

- Operation:

Consists of phase where the FTI System itself is operated and maintained in the aircraft during the FTC's. 
[Conception] FTI Measurements and Special Systems definition

The Flight-Test Instrumentation List (FTIL) is a group of measurements about what the System Engineering is planning to monitor every test. Aspects like numbering and naming standards, type and range of measurement, sampling rate and data-sourcing will be previously defined in the list. Currently Lilium Jet FTIL contains around 10K measurements created (April, 20).

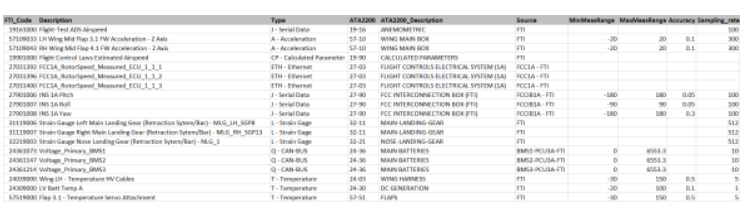

Fig. 1. Lilium FTIL sample.

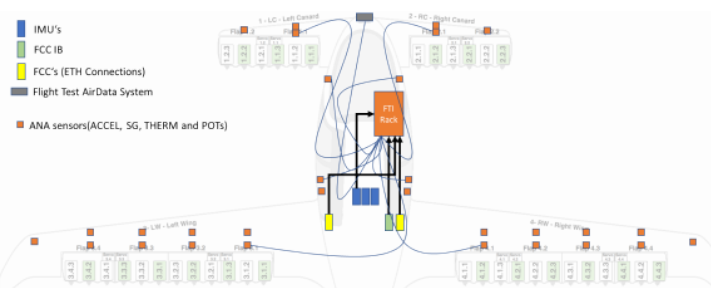

Fig. 2. Lilium Jet FTI Datasources mapping.

As FTI Special Systems we can consider a mandatory group of systems or equipment to perform the tests accordingly like Data Acquisition System (DAS), Radio-Frequency (RF) Telemetry System (Data + Image), Ground Control System (GCS), Pilot and Engineering Displays, etc.. - which provides to the FT Crew and Engineering monitoring and controllability conditions during the tests.

\section{[Conception] FTI Architecture definition}

Combining all measurements, special systems and operation requirements we can define the FTI System Architecture, as you can see in the figure below:

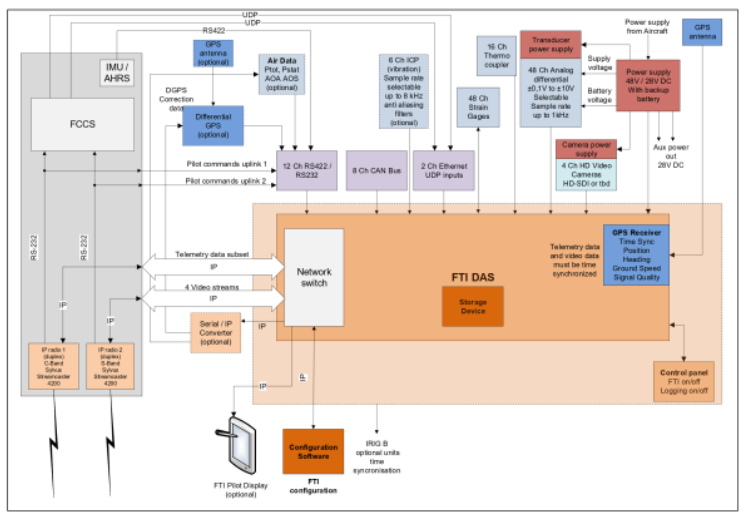

Fig. 3. Lilium FTI Airborne System Architecture.

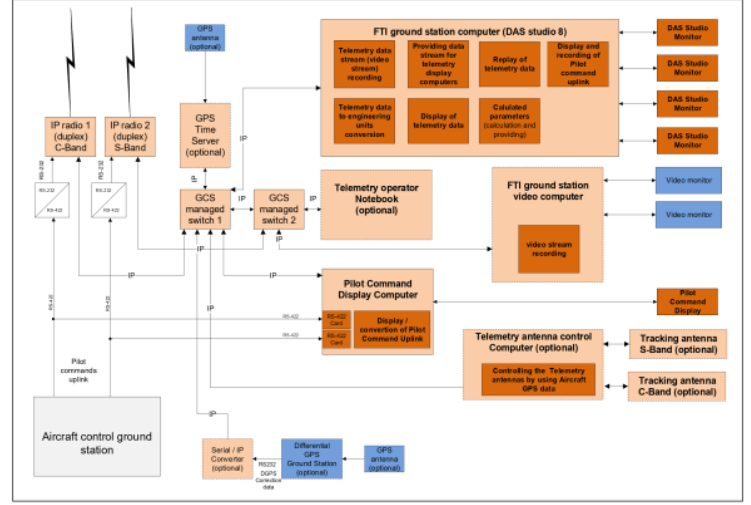

Fig. 4. Lilium FTI GCS Architecture.

\section{[Project] FTI System installation design}

The FTI System installation design includes all electrical and mechanical installation drawings. In special, the FTI Rack installation design is shown below. Inside it, we'll install the DAS, Video and Radio Telemetry Systems, as many other important FTI components:

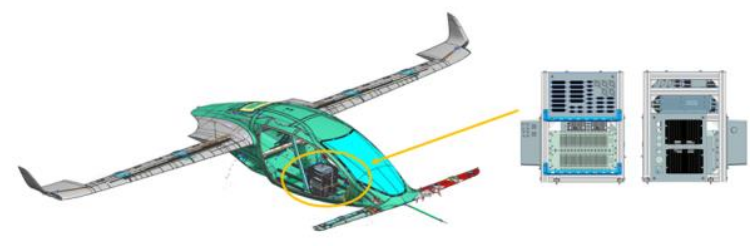

Fig. 5. Lilium Jet FTI Rack mechanical installation design.

\section{[Project] FTI Sensors installation design}

In the same way and standard, all sensors and signal sources are identified in the installation drawings, as you can see below:

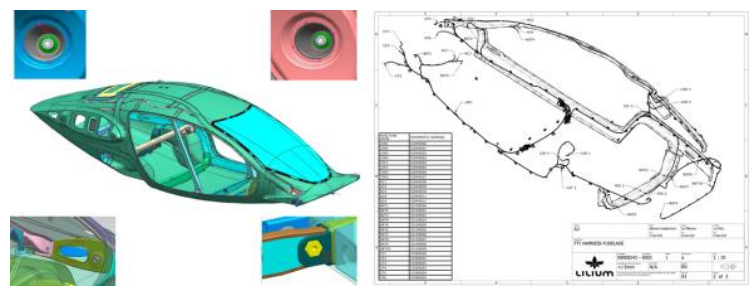

Fig. 6. Lilium FTI harnesses installation design.

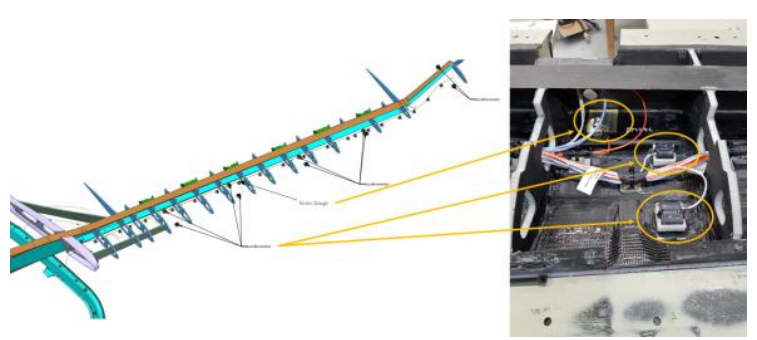

Fig. 7. Lilium FTI sensors installation design. 
As part of the $\mathrm{FTI}$ sensors documentation, is also mandatory to prepare the FTI wiring diagrams, where all electrical connections between DAS and Sensors are described, as you can see in the figure below:

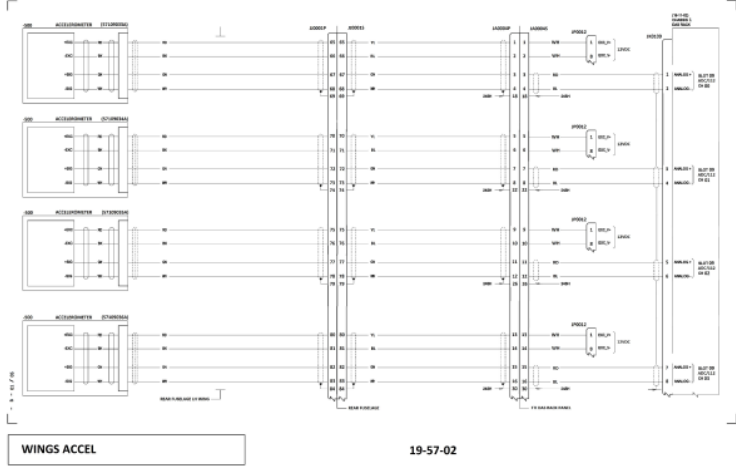

Fig. 8. Lilium FTI sensor wiring diagram example.

\section{[Integration] FTI Integration, Bench Tests and Sensors Calibration}

All Lilium Jet FTI Integration has been performed in the Software/Hardware in the Loop Laboratory (SiL and HiL), simulating flight conditions and all signal types from different data sources into the FTI DAS. In parallel, all Ground Control logical functions from the aircraft side could be verified from the Pilot Control, also available in the room, as you can see in the figure below:

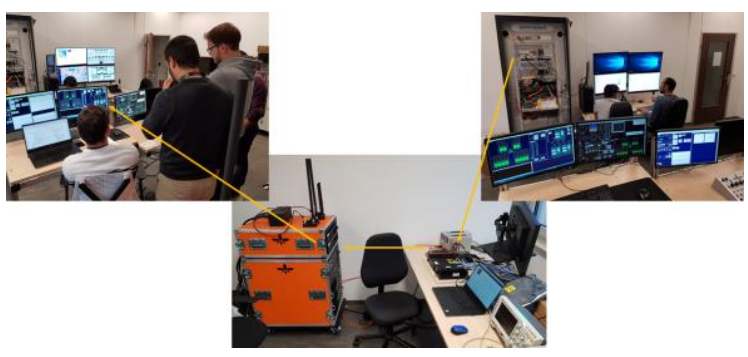

Fig. 9. Lilium FTI SiL and HiL integration tests.

About FTI Calibration process, we are following and complying with ISO-17025 requirements.

\section{[Integration] Ground Control Station design}

The GCS consists in a remote station for the aircraft monitoring (and control, for unmanned flights). From there, Flight-Test Pilots and Engineers can perform different scopes of testing and evaluate the aircraft maneuverability and controllability aspects.

When performing flight-tests, the FTI data is fully recorded inside the aircraft and transmitted from the aircraft to the GCS via RF Telemetry Link; Pilot commands - from the Pilot Control
System (PIS) - are also transmitted via RF link, but in the way-around, from the GCS to the aircraft.
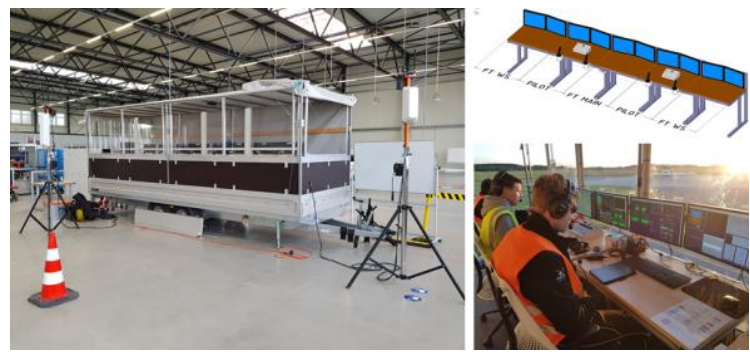

Fig. 10. Lilium GCS design and preparation.

\section{[Software Development] Lilium Flight-Test Portal development}

All information regarding to the FT Process, including $\mathrm{FTI}$, is controlled by the in-house developed FT Portal. It's a web-based application where FTIL, Reports, Test Plans and Results are controlled and registered, and can be accessed by anyone in the company via web browser.

The user-interface is friendly and the system combines web applications with desktop (scriptoriented ones) running "behind the scenes", from the user point of view.

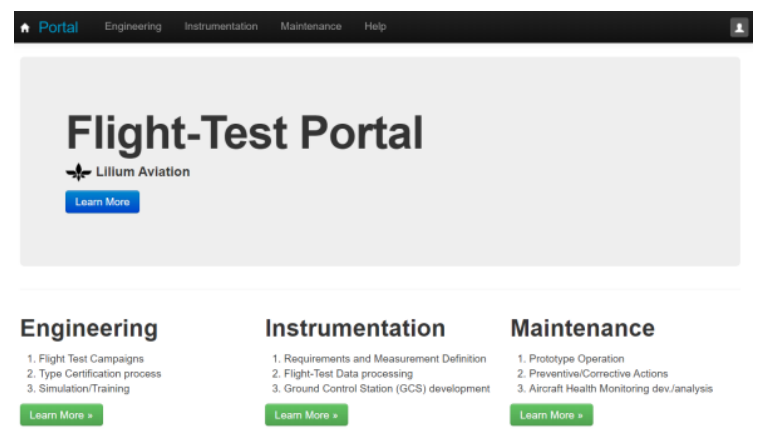

Fig. 11. Lilium FT Portal main page.

All FT Portal features are connected to the Flight-Test Database (FTDB), developed inhouse, as you can see in the figure below.

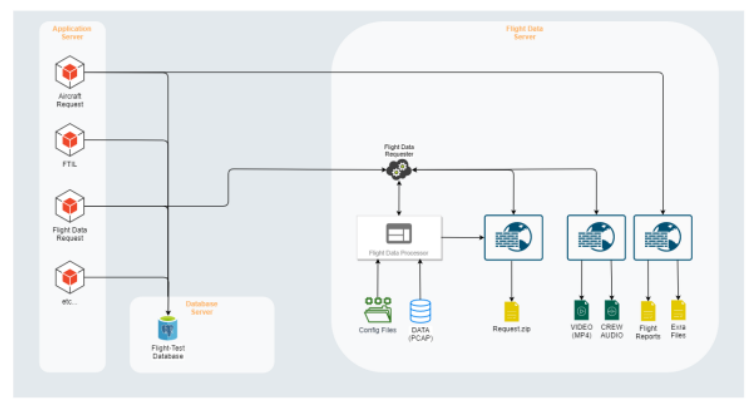

Fig. 12. Lilium FT Portal Architecture. 


\section{[Software Development] Flight-Test Results}

The Flight Test Results is a group of features that, combined, can provide all information around any test performed by Flight-Test Team to the systems engineering, which includes FT Reports and FTI processed data.

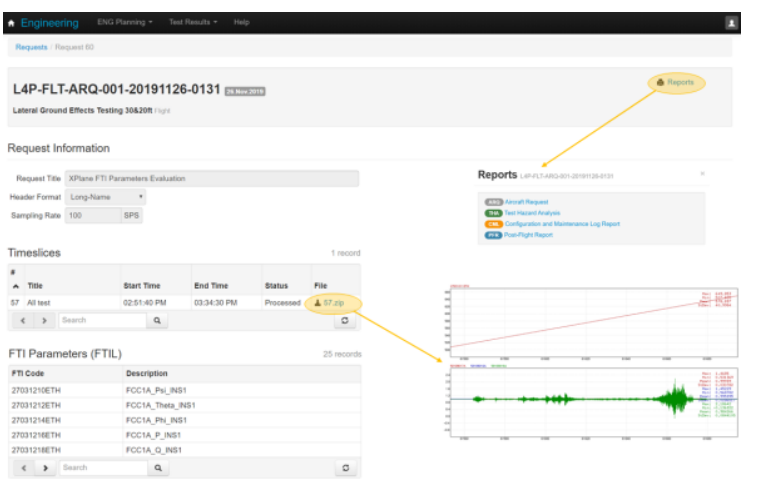

Fig. 13. Lilium FT Results user interface.

\section{[Software Development] Pilot and FTE's Displays development}

FT Displays are developed and customized inhouse for each FT Campaign, and they are available in the GCS to the Pilot and FTE's every test. Once flying unmanned, they are mandatory.

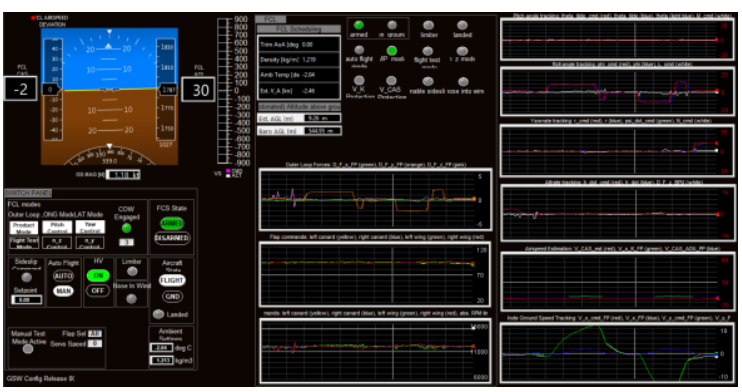

Fig. 14. Lilium Pilot and FTE's Displays (example 1).

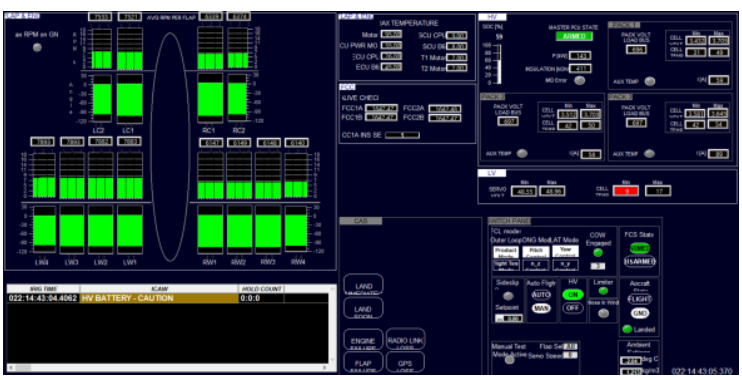

Fig. 15. Lilium Pilot and FTE's Displays (example 2).

\section{[Operation] FTI Task Management System}

All FTI tasks performed in the aircraft (valid for operation and design phases) must be traceable and logged accordingly: information like task subject, type, assignee and document reference are always important to be registered, and they can be useful for statistics analysis or even in case of incident research.

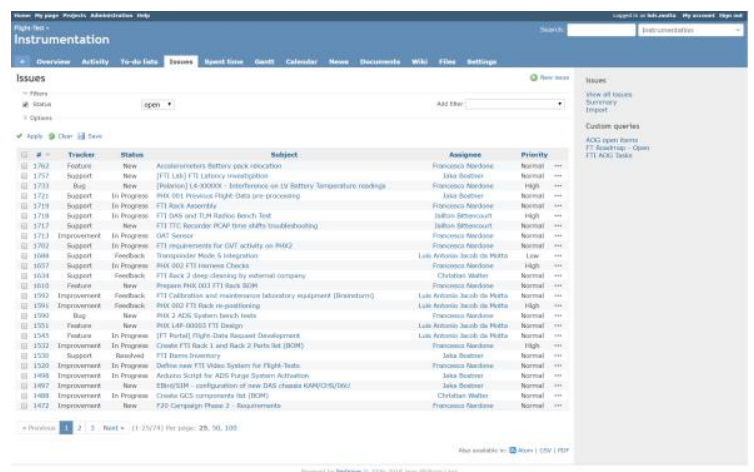

Fig. 16. FTI Tasks Management System using Redmine opensource issue tracker.

\section{[Operation] General considerations for the FTI flight preparation}

Test Plan, Risk Assessment, Configuration Control, Briefing, Pre and Post-Flight procedures are some of the mandatory safety and quality steps to be complied with when working in aviation - even more when flying unmanned.

\#SafetyFirst

During performing the FTI pre-flight procedure, systems like Data Acquisition, Data Recorder, Telemetry Radio Links and Data Processing Servers are tested and released for flight.

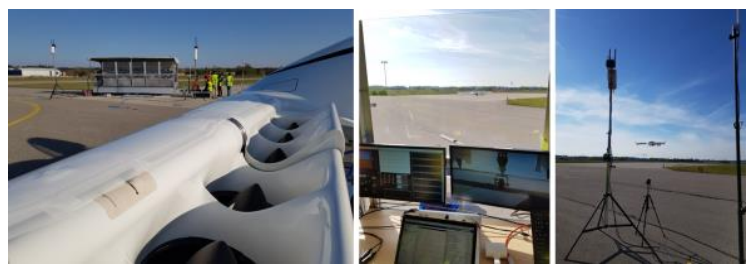

Fig. 17. Lilium Jet Flight-Test field preparation.

After performing the test, all FTI Data is moved to the FTI servers and processed accordingly converting them from a Raw Tormat to Engineering Units (e.g.: from "bits" to "Meters, degree Celsius, PSI, etc..").

The reports from the Flight Crew are also helpful, providing relevant events and details about how was the aircraft's behavior during performing maneuvers, which will support system engineering during the data analysis. 


\section{[Operation] FTI Statistics Monitoring System}

In order to improve tasks performance and/or evaluate how they are progressing, let's use the numbers in our favor. We can always correlate important Key Performance Indicators (KPI) with $\mathrm{FTI}$ planning and actions management.

FTIL growth, Design Changes rate and quantity of tests performed can give us a good expected workload indicator when performing a specific Flight-Test Campaign; or even we can calculate the average working time expended in a regular instrumentation implementation, for example.

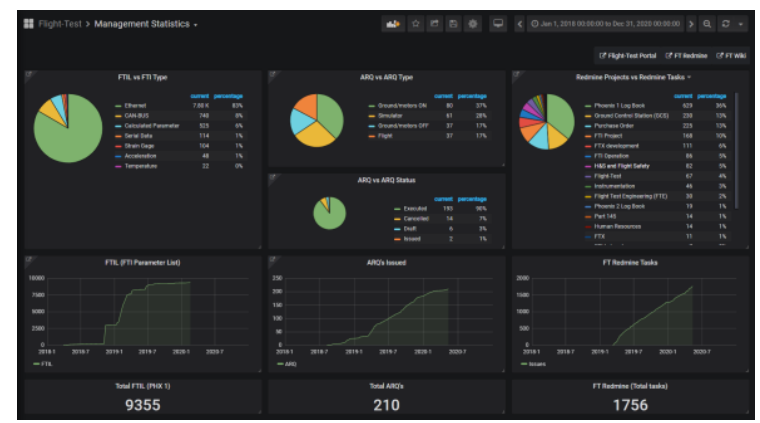

Fig. 18. FTI Statistics Dashboard using Grafana opensource interface

\section{Final Considerations}

In the end, all conventional instrumentation concepts, combined with aviation best practices and engineering experience can provide a reliable and safer test environment, increasing the quality of data acquired and test results, maximizing the aircraft systems development and mitigating issues during performing the FlightTest Campaigns. 